\title{
EDitorial Commentary \\ HAMSTRUnG: DO SEX DIFFERENCES IN HAMSTRING INJURY PROFILE NECESSITATE A DIFFERENT APPROACH TO REHABILITATION AND PREVENTION PROGRAMS IN FEMALE ATHLETES?
}

\author{
Kelly C. McInnis, DO ${ }^{1}$ \\ ${ }^{1}$ Department of Physical Medicine and Rehabilitation, Division of Sports Medicine, \\ Massachusetts General Hospital, Boston, Massachusetts USA
}

Hamstring strain injury (HSI) is one of the most common injuries encountered in running sports and can lead to significant morbidity, with time lost from participation and high rates of recurrence. Though the incidence is high in both sexes, male athletes appear to have a two to four-fold greater risk of HSI compared to female athletes, with a longer recovery. Multiple potential risk factors have been studied, and age, history of hamstring injury, ACL injury and calf injury appear to be the most significant factors in predicting index injury and recurrence. Female athletes may be relatively protected by less hamstring unit stiffness and greater muscle endurance. Other potential sex-influenced risk factors including differences in pelvic, hip and knee structural morphology and limb alignment, musculotendinous flexibility and joint mobility, and kinetic chain (primary posterior) strength imbalances require further investigation. Further research focusing both exclusively on female athlete as well as more robust comparative studies with male athletes will help us to better understand differences in HSI profile. Future investigation is necessary to determine whether a sex-specific approach to HSI rehabilitation and prevention programs will optimize clinical care for both male and female athletes.

Hamstring strain injury (HSI) is one of the most common sports-related injuries with a prevalence that appears to be increasing annually ${ }^{1}$. This injury deserves a high level of attention and investigation given the significant time lost from sports participation and high risk of injury recurrence in both male and female athletes. The risk of HSI recurrence from $16-32 \%$ is simply not acceptable 2,3 . According to a recent systematic review and meta-analysis ${ }^{4}$, the most significant risk factors for HSI are age, history of hamstring injury (Risk ratio $(R R)=2.7)$, anterior cruciate ligament (ACL) injury $(R R=1.7)$ and calf injury $(R R=1.5)$. Though sex alone does not appear to be a significant intrinsic risk factor, female athletes sustain a lower rate of HSI than male counterparts and may experience shorter recovery time and less time lost from sports participation. This has been demonstrated in several sex-comparable sports including collegiate and professional soccer and track and field ${ }^{5,6}$. Overall, like much of the sports medicine literature, females are largely underrepresented in hamstring injury studies with respect to risk factor analysis, intervention, and prevention. We need to understand the complexities and interplay of the modifiable risk factors to better care for our female and male athletes.

In their study, "Sex-based Differences in Hamstring Injury Risk Factors," O'Sullivan et $\mathrm{al}^{7}$ reviews the sex-influenced differences in intrinsic risk factors associated with HSI. The authors highlight three factors that may be protective and contribute to the discrepancy in injury rate. Women exhibit (1) increased hamstring flexibility, (2) decreased hamstring musculotendinous stiffness 
and (3) greater resistance to skeletal muscle fatigue compared to male counterparts. The authors also point out other known sex differences such as quadriceps to hamstring strength ratio and lower extremity morphologic variables (quadriceps angle, pelvic tilt) that may have an influence on HSI, with a less straightforward, logical relationship to decreased relative risk in female athletes.

The authors present an important initial investigation to add to a growing body of literature exploring sex-differences in sports injury, a critical step to promote awareness that different risk factor profiles may exist for men and women with HSI. A better understanding of modifiable factors and the complexities of their relationships will drive injury management and prevention programs. And a sexspecific component may prove essential to successful primary and secondary prevention. However, there is much work to be done in this arena and this paper underscores our gaps in knowledge. For example, we know that female athletes have both increased hamstring flexibility and generalized hip and knee joint hypermobility compared to male counterparts, but it is currently unclear how these factors influence specific lower extremity injury rates ${ }^{8}$. As the authors note, there are mixed studies regarding increased hamstring flexibility as a risk factor for hamstring injury. Green et $\mathrm{al}^{4}$ evaluated over 150 risk factors for index and recurrent HSI in sport and found that no factor related to flexibility, mobility and range of motion showed a clear relationship with risk of index HSI. In contrast, there was evidence (limited level) that hamstring muscle-tendon stiffness and reduced hamstring strength endurance, more commonly exhibited by male atheletes, are risk factors for index $\mathrm{HSI}^{4}$.

In addition, increased risk for lower extremity injuries (including ACL) has been demonstrated in collegiate female athletes with knee flexor to extensor strength ratios less than 0.759. Though hamstring strength is likely protective, women who are generally weaker in this muscle group and more quadriceps dominant, have less risk of HSI than males. Therefore, there must be more to the story. We know that female athletes more commonly exhibit a relative posterior kinetic chain weakness and hip abductor weakness that can lead to dynamic core instability ${ }^{10}$. Moreover, female athletes more commonly have a leg dominance, and this often includes asymmetric maximal hip extension, reported to increase risk of overall lower extremity injury ${ }^{11}$. Vannatta et al ${ }^{12}$ recently looked at the sex differences in gluteal muscle forces during running and found that males produced greater peak gluteus maximus force, but lesser peak hamstring force than females during running. Future directions may focus on these discrepancies in strength as well as patterns of neuromuscular control and running/sprinting biomechanics that may help to explain the relationship to HSI. The balance between core, gluteal and hamstring muscles may have a significant influence. Moreover, additional studies are needed to explain the role of muscle fatigue, which females may be more resistant, with both speed and endurance training.

The authors point out two lower extremity static/dynamic alignment factors more commonly exhibited by female athletes: quadriceps angle and anterior pelvic tilt. They may both affect the length, tension, and stiffness of the hamstring unit, but it is unclear at this time how they impact hamstring injury rate in males and females. There are several bony morphologic differences at the level of the pelvis and hip joint that may also play a role given the biarticular anatomic course of the hamstring muscle group, originating at the ischial tuberosity. Females have greater subpubic angle, intertuberosity distance and narrower ischiofemoral space than male counterparts. At the hip joint, females have a greater risk of acetabular dysplasia as well as acetabular and femoral anteversion which have been shown to cause asymmetry in maximal activation of muscles crossing the hip joint ${ }^{13}$. The articular surface area of both the hip joint and knee joint are generally less in women. And there may be differences in muscle size and architecture. It is not yet understood how these sex discrepancies in structure may contribute to hamstring injury risk and rates; another area poised for future research.

Though identification of individual risk factors for HSI is important for guiding care, we must also understand the complex and fluid interplay between factors, including the relationship to sex, to optimize management of both male and female athletes. While rates of HSI are lower in women compared to men, it is a common index and recurrent injury for both in sports 
medicine. Future research focused both exclusively on female athletes as well as comparative studies (correcting the existing disparity in sex representation) will help us to better understand whether we need more of a sex-specific approach to HSI rehabilitation, and whether our existing prevention programs should be modified for female athletes. Our investigation and understanding of sex differences in sports injury profiles and patterns must continue to grow and reflect the exponential rise of female athlete participation worldwide.

\section{Conflict of Interest Statement}

The author reports no conflict of interest with the contents of this manuscript.

\section{Corresponding Author}

Kelly C. McInnis, DO

Department of Physical Medicine \& Rehabilitation

Division of Sports Medicine

Massachusetts General Hospital

175 Cambridge St. $4^{\text {th }}$ Floor

Boston, Massachusetts 02114 USA

kcmcinnis@partners.org

\section{REFERENCES}

1. Ekstrand J, Waldén M, Hägglund M. Hamstring injuries have increased by $4 \%$ annually in men's professional football, since 2001: A 13-year longitudinal analysis of the UEFA Elite Club injury study. Br J Sports Med. 2016;50(12):731-737. doi:10.1136/BJSPORTS-2015-095359

2. Feeley BT, Kennelly S, Barnes RP, et al. Epidemiology of national football league training camp injuries from 1998 to 2007. Am J Sports Med. 2008;36(8):1597-1603. doi:10.1177/0363546508316021

3. Opar DA, Williams MD, Shield AJ. Hamstring strain injuries: Factors that Lead to injury and re-Injury. Sport Med. 2012. doi:10.2165/11594800-00000000000000

4. Green B, Bourne MN, Van Dyk N, Pizzari T. Recalibrating the risk of hamstring strain injury (HSI): A 2020 systematic review and meta-analysis of risk factors for index and recurrent hamstring strain injury in sport. $\mathrm{Br} J$ Sport Med. 2020;54:1081-1088. doi:10.1136/bjsports-2019-100983

5. Cross KM, Gurka KK, Saliba S, Conaway M, Hertel J. Comparison of hamstring strain injury rates between male and female intercollegiate soccer athletes. Am J Sports Med. 2013;41(4):742-748. doi:10.1177/0363546513475342

6. Larruskain J, Lekue JA, Diaz N, Odriozola A, Gil SM. A comparison of injuries in elite male and female football players: A five-season prospective study. Scand J Med Sci Sport. 2018;28(1):237-245. doi:10.1111/sms.12860

7. O'Sullivan, Lucy; Tanaka M. Sex-based differences in hamstring injury risk factors. J Women's Sport Med. 2021;1(1).

8. Pacey V, Nicholson LL, Adams RD, Munn J, Munns CF. Winner of the 2009 systematic review competition: Generalized joint hypermobility and risk of lower limb joint injury during sport: A systematic review with meta-analysis. Am J Sports Med. 2010;38(7):14871497. doi: $10.1177 / 0363546510364838$

9. Knapik JJ, Bauman CL, Jones BH, Harris JM, Vaughan L. Preseason strength and flexibility imbalances associated with athletic injuries in female collegiate athletes. Am J Sports Med. 1991;19(1):76-81. doi:10.1177/036354659101900113

10. Huston LJ, Wojtys EM. Neuromuscular performance characteristics in elite female athletes. Am J Sports Med. 1996;24(4):427-436. doi:10.1177/036354659602400405

11. Nadler SF, Malanga GA, DePrince M, Stitik TP, Feinberg JH. The relationship between lower extremity injury, low back pain, and hip muscle strength in male and female collegiate athletes. Clin J Sport Med. 2000;10(2):89-97. doi:10.1097/00042752200004000-00002

12. Vannatta CN, Kernozek TW. Sex differences in gluteal muscle forces during running. Sport Biomech. 2021;20(3):319-329. doi:10.1080/14763141.2018.1548641

13. Nyland J, Kuzemchek S, Parks M, Caborn DNM. Femoral anteversion influences vastus medialis and gluteus medius EMG amplitude: Composite hip abductor EMG amplitude ratios during isometric combined hip abduction-external rotation. I Electromyogr Kinesiol. 2004;14(2):255-261. doi:10.1016/S1050-6411(03)00078-6 